\title{
Phénomènes dissipatifs dans une digue à paroi perforée
}

\author{
Jean-Marc Rousset \\ Doctorant, M2C-Groupe Mécanique des Fluides, UMR 6143, Université de Caen \\ Tanja Kohl \\ Elève Ingénieur, LWI, Université Technique de Brünswick (Allemagne) \\ Michel Bélorgey \\ Professeur, M2C-Groupe Mécanique des Fluides, UMR 6143, Université de Caen
}

\section{Résumé}

La capacité de réflexion d'un caisson Jarlan est étudiée expérimentalement en canal. Les essais ont été réalisés avec des houles spectrales et régulières ainsi que pour deux largeurs de bermes. Nous observons en particulier une décomposition spectrale de la houle régulière incidente dans la gamme de périodes où le caisson est le moins réfléchissant. Parallèlement nous mettons en évidence un phénomène de déversement lors du passage de l'écoulement à travers les perforations et donit les capacités dissipatrices d'énergie sont liées à la hauteur de la houle.

\begin{abstract}
The reflection ability of a Jarlan caisson is analysed from tests in a wave flume. Both random and regular waves were generated and two berm widths were tested. A spectral dispersion of reflected regular waves is observed especially for periods of caisson low reflection. In the same time, we show that spilling phenomenon occurs due to the flow through the perforated wall and that its dissipating ability is linked to the wave height.
\end{abstract}

\section{Introduction}

Les digues à parois perforées sont reconnues pour engendrer une faible réflexion de la houle incidente. Cette caractéristique est appréciée lorsque les quais doivent être peu réfléchissants afin de limiter l'agitation dans les bassins portuaires. Ces digues perforées se retrouvent aussi dédiées à la protection de ports de commerce en tant que brise-lames extérieur.

La dissipation de l'énergie incidente peut être appréciée par la détermination du coefficient de réflexion de l'ouvrage. D'une façon générale, les modèles analytiques et numériques sont confrontés à la complexité des phénomènes nonlinéaires engendrés par ces parois perforées. Il nous est apparu utile de mettre en évidence expérimentalement certains paramètres physiques qui peuvent 
contribuer à l'efficacité d'une telle digue mais qui sont parfois difficilement appréciés dans les simulations numériques.

\section{Description des essais}

\subsection{Description de la maquette}

La maquette testée est la reproduction selon la similitude de Froude et au $25^{\text {eme }}$, d'un caisson Jarlan de la digue de Dieppe (Seine-Maritime). La largeur de la chambre B est de $540 \mathrm{~mm}$ et l'épaisseur de la paroi perforée est de $40 \mathrm{~mm}$. Les perforations sont cylindriques, de diamètre $40 \mathrm{~mm}$ et contribuent à une porosité globale de $28 \%$. La partie inférieure de la chambre est pleine et simule le lest de béton du caisson prototype (Rousset et Bélorgey, 1998).

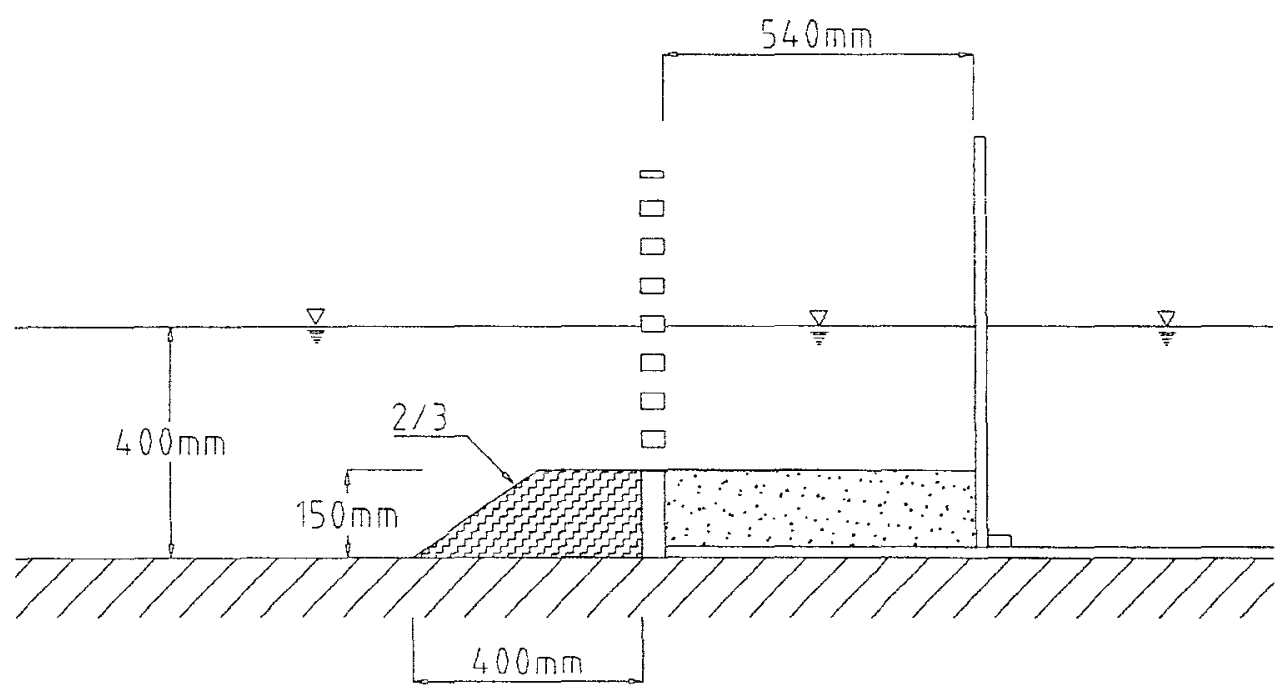

Fig. 1 : Dimensions de la maquette et de la berme courte.

Model and narrow berm dimensions.

\subsection{Description des bermes}

Nous avons mis en œuvre deux bermes différentes. Leur carapace est composée d'enrochements simulant un poids de 3 à 4 tonnes en nature, comme les éléments utilisés à Dieppe. Les couches intermédiaires sont constituées de blocs de poids décroissants. Par ailleurs, la maquette reposant directement sur le fond du canal, le massif de sous-bassement du caisson n'est pas reproduit.

De même hauteur $(15 \mathrm{~cm})$ et de pente de talus identique $(2 / 3)$, les deux bermes different cependant par leur largeur. La berme dite courte est large de $40 \mathrm{~cm}$ (Fig. 1), celle dite moyenne de $64 \mathrm{~cm}$.

\subsection{Conditions des essais}

Les essais ont été réalisés dans un canal à houle long de $22 \mathrm{~m}$ et large de $0,80 \mathrm{~m}$. La maquette est placée à 20 mètres du batteur, directement sur le fond plat d'un canal, la profondeur d'eau étant constante et de $0,40 \mathrm{~m}$. 
Les houles monochromatiques ont été générées pour des périodes $T$ variant de 1,1 à $5,0 \mathrm{~s}$, les hauteurs variant de 5 à $15 \mathrm{~cm}$. Les houles spectrales (PiersonMoskowitz) avaient des périodes de pic $T_{p}$ égales à $1,4,1,6,1,8$ et $2,4 \mathrm{~s}$, les hauteurs significatives étant comprises entre 3,5 et $13 \mathrm{~cm}$.

La détermination des caractéristiques des houles incidente et réfléchie est obtenue par la méthode à trois sondes décrite par Mansard et Funcke (1980), avec la première sonde disposée à une distance constante de $10 \mathrm{~m}$ du batteur. Pour chaque essai, le traitement des données a porté sur un minimum de 500 vagues, la fréquence d'échantillonnage étant adaptée afin d'assurer la meilleure résolution spectrale.

\section{Présentation et analyse des résultats}

\subsection{Influence de la berme sur le coefficient de réflexion}

Sur la Fig. 2 sont présentés le coefficient de réflexion moyen de la maquette du caisson Jarlan, lors des essais en houle régulière avec chacune des deux bermes. Ce coefficient représente le rapport de l'énergie réfléchie sur l'énergie incidente déterminées par les moments d'ordre zéro. En abscisse se trouve la largeur relative $\mathrm{B} / \mathrm{L}$ ', L' étant la longueur d'onde locale calculée pour la profondeur de la chambre de dissipation et la période de la houle ( $\mathrm{T}$ ou $\mathrm{T}_{\mathrm{p}}$ dans la houle aléatoire).

Il apparaît que le minimum de réflexion est atteint pour un rapport $B / L$ ' proche de 0,16 (Fig. 2). Les résultats provenant d'essais réalisés sur la même maquette mais sans berme et sans lest, soit une profondeur de la chambre identique à celle devant l'ouvrage (Bélorgey et al, 1999), montrent un $\mathrm{Cr}$ minimum pour une valeur B/L' similaire. L'obtention d'une réflexion minimale semble donc être liée principalement à la porosité et à la largeur $\mathrm{B}$ de la chambre.

Dans la plage $0,16<\mathrm{B} / \mathrm{L}^{\prime}<0,20$ en houle régulière, le Cr mesuré avec une berme varie entre 30 et $40 \%$ alors qu'il n'est que de $20 \%$ sans berme et sans lest. Les mesures en houle aléatoire présentent également une réflexion minimale supérieure à $40 \%$ (Tableau 1). Il semble donc que la diminution de la profondeur locale, engendrée par la présence de la berme et du lest, entraîne un net accroissement de la réflexion.

La figure 2 et le tableau 1 montrent de surcroît que la berme courte est associée à une réflexion globale légèrement plus forte que la berme moyenne. Pour des conditions de houle strictement identiques (hauteur et période), les écarts de $\mathrm{Cr}$ varient entre 10 et $20 \%$, valeurs qui laissent supposer que les enrochements ne dissipent qu'une faible partie de l'énergie incidente. 


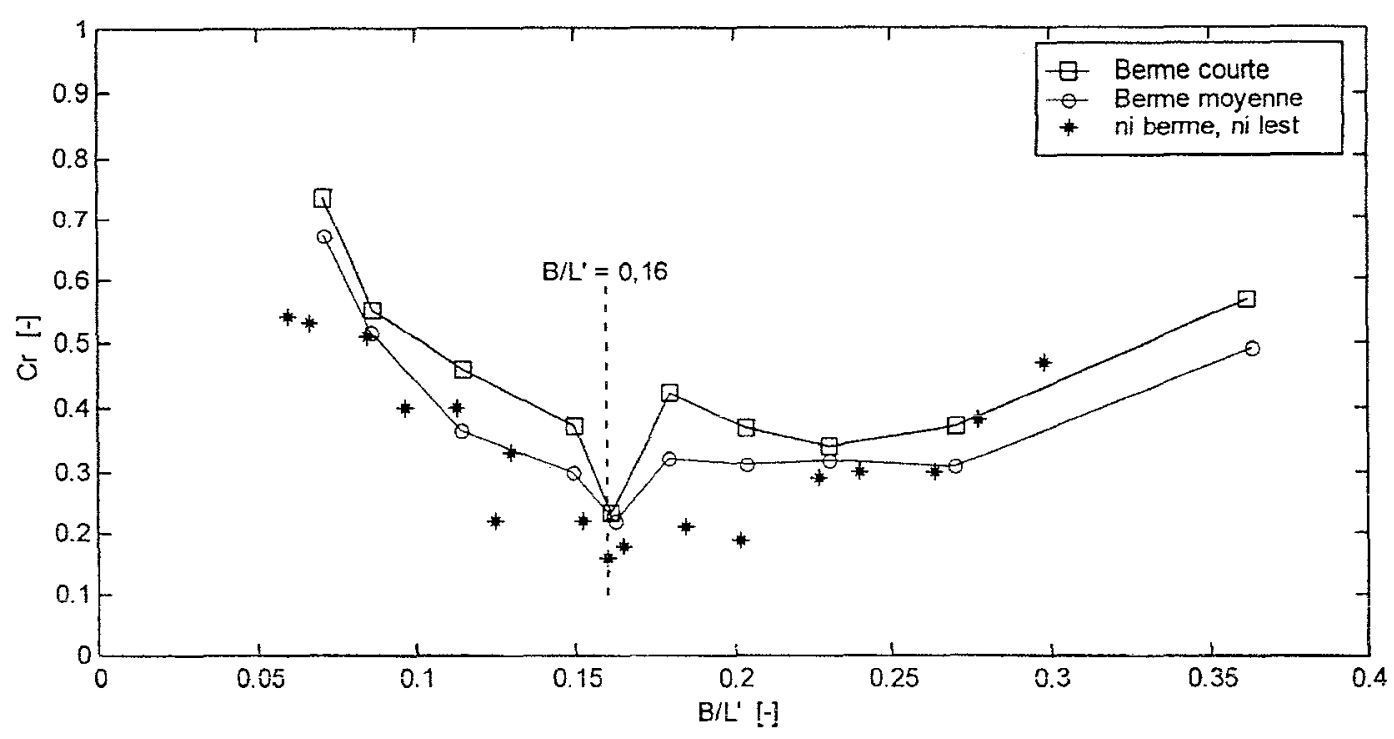

Fig. 2 : Coefficients de réflexion moyens en houle régulière.

Mean reflection coefficients under regular waves condition.

\begin{tabular}{|c|l|c|c|c|c|}
\cline { 2 - 6 } \multicolumn{1}{c|}{} & \multicolumn{1}{c|}{ B/L' $[-]$} & 0,27 & 0,23 & 0,20 & 0,15 \\
\hline \multirow{2}{*}{$\operatorname{Cr}[-]$} & Berme courte & 0,64 & 0,56 & 0,47 & 0,40 \\
\cline { 2 - 6 } & Berme moyenne & 0,54 & 0,45 & 0,41 & 0,41 \\
\hline
\end{tabular}

Tableau 1 : Coefficients de réflexion moyens en houle aléatoire. Mean reflection coefficients under random waves condition.

\subsection{Influence de la hauteur de houle incidente}

Sur la Fig. 3 sont reportés les coefficients de réflexion pour les deux bermes et deux hauteurs de houle régulière $\mathrm{Hi}=0,05 \mathrm{~m}$ et $\mathrm{Hi}=0,10 \mathrm{~m}$.

Dans la gamme $0,20<\mathrm{B} / \mathrm{L}^{\prime}<0,35$, $\mathrm{Cr}$ décroît alors que la hauteur de houle augmente, ceci pour les deux bermes. Les essais en houle spectrale, dont les résultats sont donnés dans le Tableau 1, présentent la même caractéristique : une hauteur de houle croissante provoque une décroissance de la réflexion de l'ouvrage.

Lorsque $\mathrm{B} / \mathrm{L}$ ' est compris entre 0,15 et 0,20 , il est difficile de dégager une tendance commune aux deux bermes. Une berme moyenne semble donner une réflexion proche de $30 \%$ quelle que soit la hauteur de houle, sauf pour $B / L^{\prime}=0,16$ où le minimum est atteint avec $20 \%$.

Une berme courte montre une réponse plus complexe. La valeur minimale de $\mathrm{Cr}$ est la même pour les deux hauteurs de houle $(24 \%)$ et pour $B / L^{\prime}=0,16$. Toutefois de part et d'autre de cette valeur de $B / L$ ', Cr augmente brusquement jusqu'à $45 \%$ lorsque la hauteur de houle croît. 

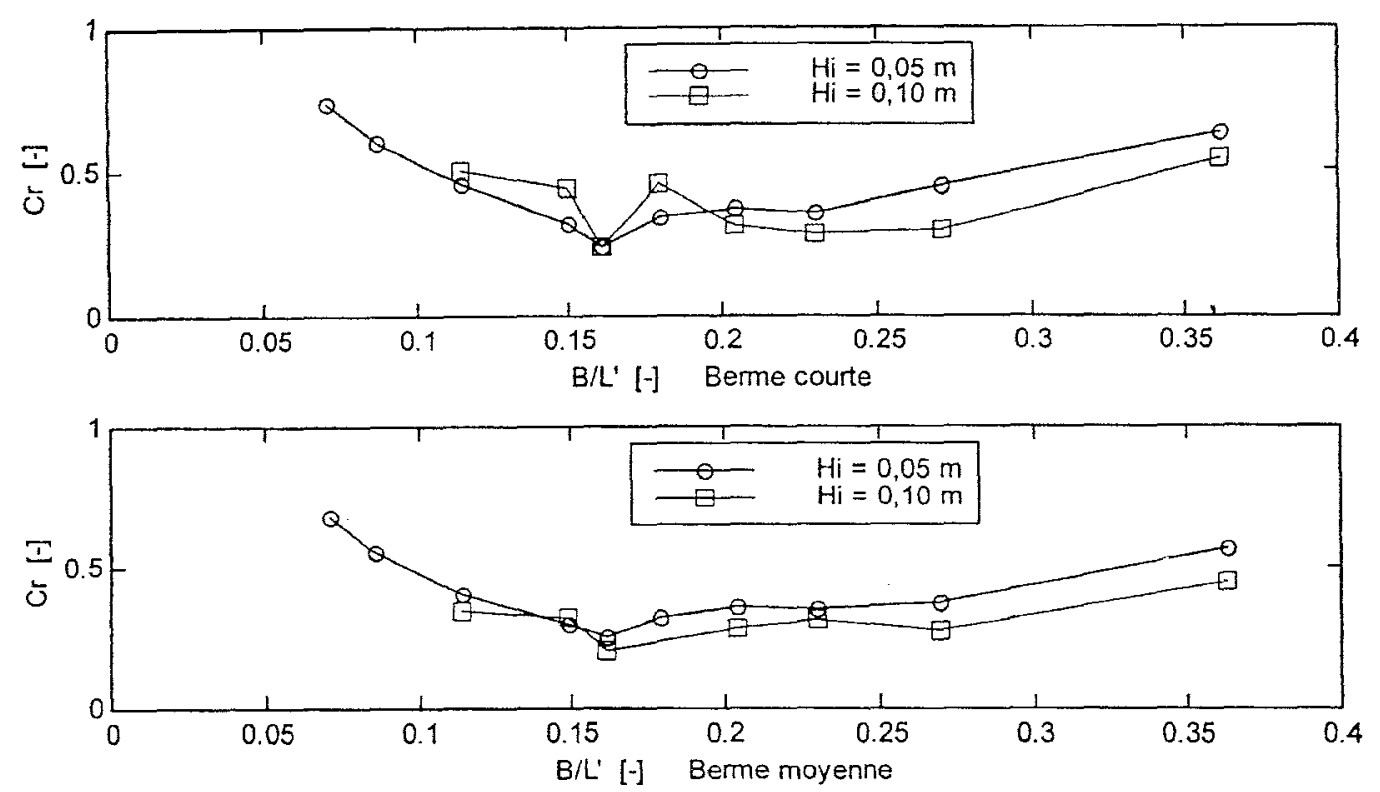

Fig. 3 : Evolution du $\mathrm{Cr}$ avec deux hauteurs de houle régulière. Evolution of $\mathrm{Cr}$ with two regular wave heights.

Deux commentaires peuvent donc être avancés au vu de ces résultats. Le premier point est la mise en évidence de la hauteur de houle incidente comme paramètre induisant une plus faible réflexion de l'ouvrage. La partie 3.4 présentera d'ailleurs un des mécanismes expliquant cette observation.

Le deuxième point concerne le type de berme à envisager lors de la conception de la digue. Une berme moyenne semble procurer une plus faible réflexion de la houle qu'une berme courte habituellement employée. Cette solution présente toutefois un handicap dans le volume d'enrochements nécessaire à sa mise en place.

\subsection{Décomposition spectrale}

Sans phénomène de franchissement et en houle régulière, nous avons déterminé le coefficient de réflexion de l'ouvrage selon deux méthodes. La première correspond au calcul des moments spectraux pour le spectre comprenant au minimum la fréquence fondamentale et les deux premiers harmoniques (résultats précédemment présentés). Le second calcul du coefficient de réflexion est basé sur le rapport de l'amplitude de la houle réfléchie sur celle incidente, uniquement pour la fréquence fondamentale. La Fig. 4 présente la comparaison de ces deux méthodes pour les essais avec une houle incidente régulière de hauteur $\mathrm{Hi}=0,10 \mathrm{~m}$.

L'allure de la variation du $\mathrm{Cr}$ calculé uniquement sur le fondamental est très régulière au contraire des résultats sur le spectre complet. En dehors de la gamme d'efficacité du caisson ( $B / L^{\prime}<0,15$ et $\left.B / L^{\prime}>0,25\right)$, les coefficients de réflexion obtenus par les deux méthodes sont très proches. Nous pouvons donc considérer que l'onde réfléchie est de même période que l'onde incidente. 


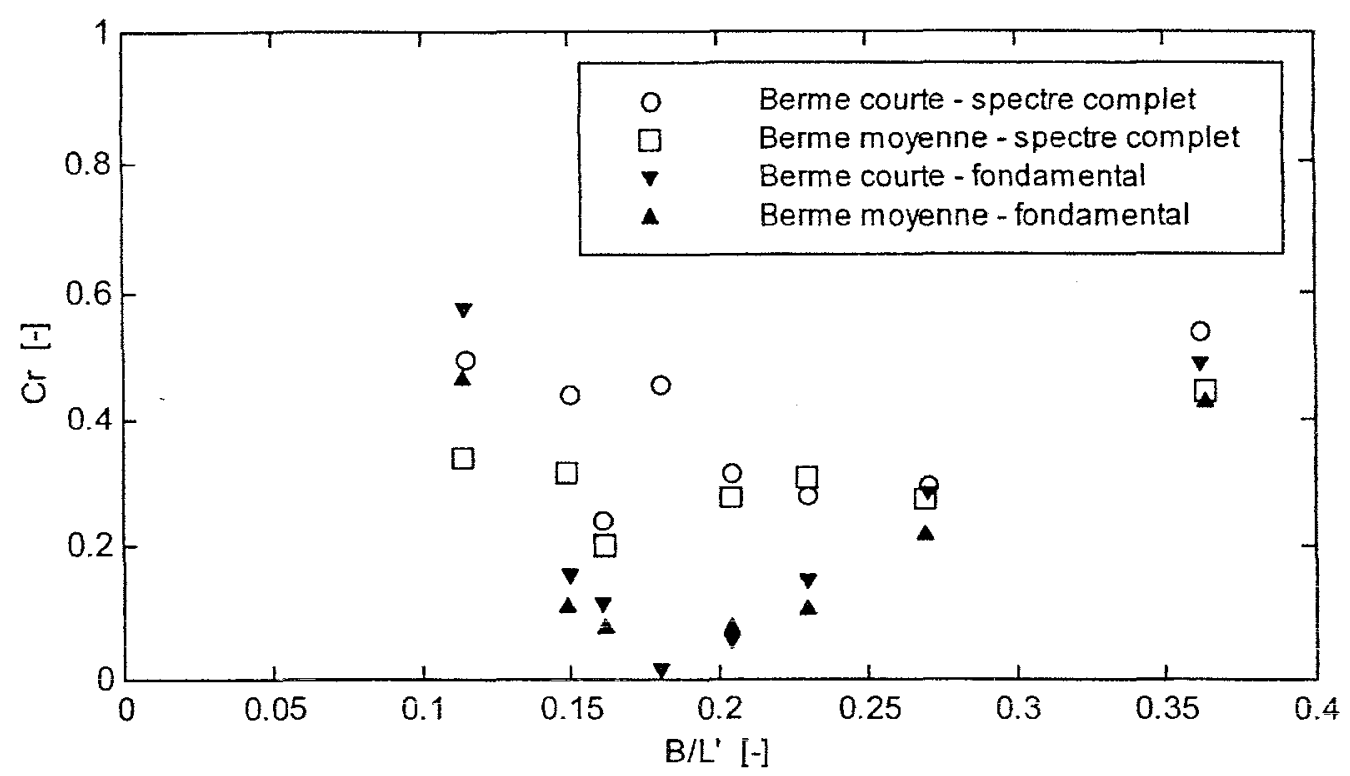

Fig. 4 : Comparaison des $\mathrm{Cr}$ calculés sur le spectre et sur le fondamental.

Comparison between $\mathrm{Cr}$ based on whole spectra and foundamental frequency.

Quand $\mathrm{B} / \mathrm{L}$ ' est compris entre 0,15 et 0,25 , la méthode basée sur le fondamentai présente un $\mathrm{Cr}$ minimum de $5 \%$ pour un $\mathrm{B} / \mathrm{L}^{\prime}$ de $0,18(\mathrm{~T}=2 \mathrm{~s})$. En ne prenant en compte que sa période principale, la houle incidente semble donc presque entièrement absorbée par le caisson.

En fait dans cette même gamme de $\mathrm{B} / \mathrm{L}$ ', le coefficient de réflexion global est assez fort, compris entre $20 \%$ environ $\left(B / L^{\prime}=0,16\right)$ et $45 \%\left(B / L^{\prime}=0,18\right)$. Il existe donc, non pas une réelle dissipation de l'énergie incidente, mais plutôt un transfert d'une grande partie de cette énergie, de la fréquence fondamentale vers le premier harmonique.

La houle est par conséquent décomposée spectralement par le caisson Jarlan et l'onde réfléchie voit son énergie portée essentiellement par la fréquence double de celle de l'onde incidente. La maîtrise de ce phénomène de transfert d'énergie pourrait conduire à une dissipation plus importante de l'énergie de la houle réelle et ainsi à une optimisation de la conception des digues à paroi perforée.

\subsection{Ecoulement à travers les perforations}

Les fluctuations de la surface libre à proximité des différentes parois du caisson et dans la chambre de dissipation ont été mesurées par 7 limnigraphes. Complétées par un suivi des oscillations par une caméra vidéo, ces mesures ont permis de schématiser sur la Fig. 5 les écoulements lors des phases d'ascension et de retrait des vagues.

Lors de la montée de la vague sur la paroi perforée, le niveau d'eau dans la chambre, de l'autre côté de cette paroi, est identique au niveau moyen au repos. Si la hauteur de houle incidente augmente, la crête va passer à travers les perforations de plus en plus hautes et retomber dans le caisson en une multitude de cascades simultanées. 


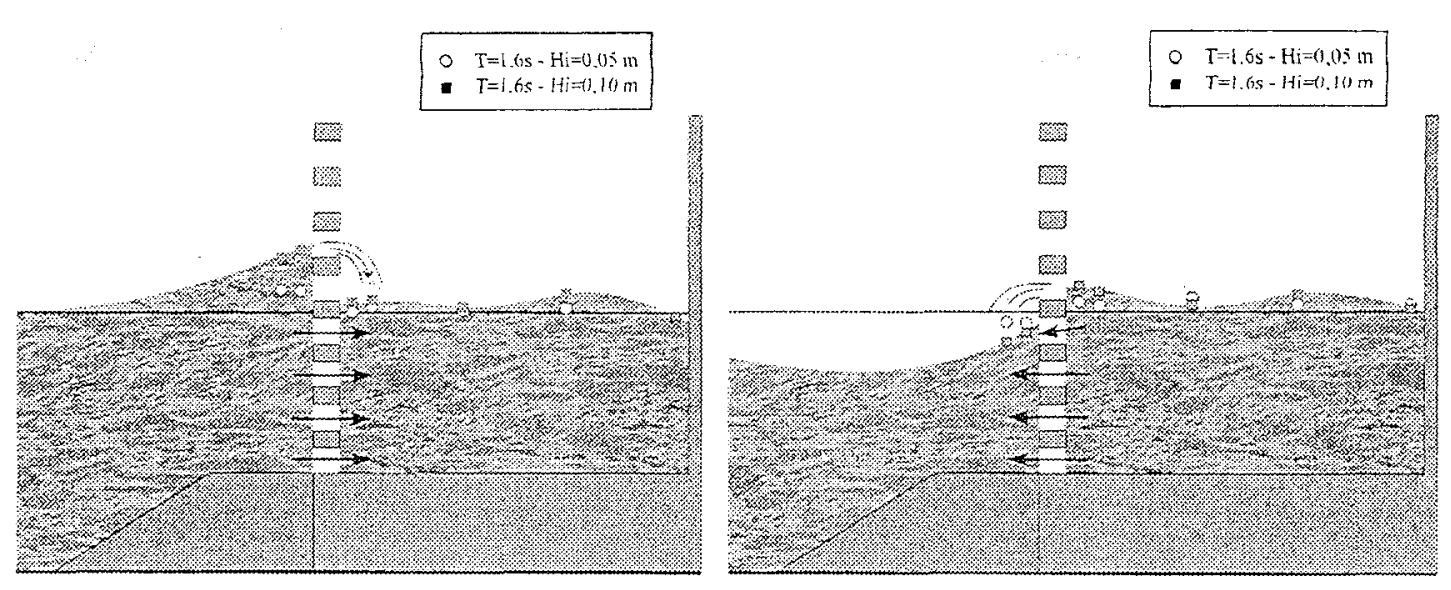

Fig. 5 : Déversements lors de l'ascension et du retrait des vagues. Spills due to the crest and and the trough of waves.

Lors du retrait de la vague, le niveau d'eau devant la paroi perforée devient inférieur au niveau au repos alors que du côté de la chambre une ascension se produit. Là encore le déversement de l'eau du caisson vers l'extérieur provoque de forts remous qui contribuent à la dissipation de l'énergie de l'écoulement. Celle-ci est d'autant importante que la hauteur de chute est importante, c'est à dire que la hauteur de la houle incidente augmente.

Le creux et la crête d'une vague générant un déversement, sa période d'occurrence est égale à la moitié de la période de la houle. Nous pouvons donc supposer qu'il contribue à la prédominance du premier harmonique précédemment mentionné, notamment quand le caisson est le moins réfléchissant $\left(B / L^{\prime}\right.$ entre 0,15 et 0,25$)$.

Toutefois la nature des perforations pourrait être un élément prépondérant dans l'apparition de ce type de dissipation. Des fentes horizontales ou des trous cylindriques semblent en effet favoriser ce phénomène de cascades. Une répartition adéquate de ces perforations pourrait de plus favoriser cette dissipation. Il nous reste cependant à étudier des parois à fentes verticales, analogues à celles utilisées au Japon (Tâniñioto et Takahashi, 1994), pour valider notre hypothèse.

\section{Conclusions préliminaires}

L'analyse expérimentale de la réflexion d'un caisson Jarlan avec sa berme montre que son fonctionnement hydraulique est complexe et que les phénomènes du second ordre ne sont pas négligeables.

Une faible réflexion de la structure est obtenue avec l'augmentation de la hauteur de la houle incidente, l'énergie étant alors en partie dissipée par des déversements à travers les perforations supérieures.

De plus la mise en évidence d'un transfert d'énergie de la fréquence fondamentale vers le premier harmonique démontre que la conception du caisson Jarlan peut encore évoluer pour devenir plus dissipatif. 
Des études supplémentaires sont par conséquent nécessaires afin de maîtriser les effets de second ordre engendrés par l'écoulement, en fonction de la porosité de l'écran, de la profondeur devant l'ouvrage et dans la chambre du caisson.

\section{Bibliographie}

Bélorgey M., Rousset JM., Tabet-Aoul EH, Bergmann H., de Gerloni M., Colombo D., Franco L. et Passoni G., 1999. "Perforated caisson breakwaters: wave loads and hydraulic performance", Proc. of Int. Conf. on Coastal Structures, Santander (Spain), Ed. Balkema, pp 603-612.

Mansard, E.P.D. et Funke, E.R. (1980). "The Measurement of Incident and Reflected Spectra Using a Least Square Method", Proc. 17th Coastal Engineering Conf., Sidney, pp 154-172.

Rousset, J.-M. et Bélorgey, M. (1998). "Analyse des variations de pressions générées par la houle sur la digue Jarlan de Dieppe", Vèmes Journées Nationales Génie civil - Génie côtier, Paralia Ed., pp 223-230.

Tanimoto, K. et Takahashi, S. (1994). "Design and construction of caisson breakwaters - the Japanese experience", Coastal Eng. "Special Issue on Vertical Breakwaters", Vol 22, No 1/2, pp 57-77. 